\title{
Zervikale Rückenmarkverletzungen bei vorbestehender Spinalkanalstenose
}

\author{
Chris Schulz, René Mathieu, Uwe Max Mauer
}

$\begin{array}{ll}\text { ABKÜRZUNGSVERZEICHNIS } \\ \text { ACCF } & \text { Anterior Cervical Corpectomy and Fusion } \\ \text { ACDF } & \text { Anterior Cervical Discectomy and Fusion } \\ \text { ASIA } & \text { American Spinal Injury Association } \\ & \text { Impairment Scale } \\ \text { DTI } & \text { Diffusion Tensor Imaging } \\ \text { ISP } & \text { Intraspinal Pressure } \\ \text { MEP } & \text { motorisch evozierte Potenziale } \\ \text { OLF } & \text { Ossification of the Ligamentum flavum } \\ \text { OPLL } & \text { Ossification of the posterior longitudinal } \\ & \text { Ligament } \\ \text { SCI } & \text { Spinal Cord Injury } \\ \text { SCIWORET Spinal Cord Injury without radiological } & \text { Evidence of Trauma } \\ \text { SCPP } & \text { Spinal Cord Perfusion Pressure } \\ \text { SLIC } & \text { Subaxial Cervical Spine Injury Classifica- } \\ & \text { tion } \\ \text { SSEP } & \text { somatosensibel evozierte Potenziale } \\ \text { zRMV } & \text { zervikale Rückenmarkverletzung } \\ \text { zSKS } & \text { zervikale Spinalkanalstenose }\end{array}$

\section{Hintergrund}

Die zervikale Rückenmarkverletzung (zRMV) ist eine extrem lebensqualitätseinschränkende, komplikationsträchtige und kostenintensive Traumafolge. Betroffene Patienten und deren Behandler kommen in der Akutsituation nicht selten in ein therapeutisches Dilemma, das sich aufgrund der aktuell verfügbaren Datenlage zu den Behandlungsergebnissen nur schwer auf hohem Evidenzniveau auflösen lässt. Die aktuelle AO-Spine-SLIC-Klassifikation (SLIC: Subaxial Cervical Spine Injury Classification) sieht bei zusätzlich zur zRMV vorliegender zervikaler Spinalkanalstenose (zSKS) den Verstärker „ " “ (bei fortbestehender Kompression neurogener Strukturen und inkompletter neurologischer Störung) sowie die Modifikatoren „M2“ (bei relevanter Bandscheibenherniation) und „M3“ (bei OPLL und OLF) vor [1]. Damit wird das negative prädiktive Potenzial einer begleitenden kompressionsrelevanten degenerativen Spondylopathie bei der zRMV unterstrichen.

Mit der MRT existiert zwar ein Diagnostikum, welches über das Ausmaß einer zSKS und den Umfang einer zRMV recht gut informieren kann. Jedoch lässt sich aus dem
MRT allein keine zweifelsfreie Behandlungsentscheidung ableiten. Diverse chirurgische Optionen existieren für die zSKS und die zRMV, aber wir wissen weiterhin nicht mit hoher Sicherheit, welche invasive Option zu welchem Zeitpunkt die beste ist. Die komplexen zellulären und molekularen Veränderungen, die zu einer klinischen Verschlechterung einer zSKS führen, sind ebenso wenig ausreichend verstanden, wie es die Erholungsmechanismen nach einer Rückenmarkverletzung sind. Demzufolge sind auch die pathophysiologischen Grundlagen für neuroprotektive Therapiekonzepte schwierig zu begründen und zu implementieren.

Der nachfolgende Übersichtsartikel soll die aktuellen Empfehlungen aus prospektiven Studien und systematischen Reviews zusammenfassen.

\section{Epidemiologie}

Die zSKS ist in der erwachsenen Bevölkerung der westlichen Industrienationen weit verbreitet, speziell mit zunehmendem Lebensalter ( $>75 \%$ der $>65$-jährigen zeigen im MRT eine zSKS). In den meisten dieser Fälle (speziell im Initialstadium) bestehen jedoch erhebliche Diskrepanzen zwischen Bildgebung und neurologischer Symptomatik. Nicht selten wird jedoch selbst asymptomatischen Patienten empfohlen, eine zSKS prophylaktisch operieren zu lassen, da ein erhöhtes Risiko für eine unfallbedingte zRMV bestünde (ca. 50\% der SKS-Pat. werden vermutlich entsprechend beraten [2]). HWS-Verletzungen sind zwar eine typische Unfallfolge, die Häufigkeit einer zRMV (in Zusammenhang mit Unfällen aller Art und Schwere) liegt jedoch bei unter $1 \%[2,3]$. In 10-15\% der zRMV-Fälle finden sich keine Hinweise für knöcherne oder diskoligamentäre Verletzungen, was als SCIWORET (Spinal Cord Injury without radiological Evidence of Trauma) bezeichnet wird [4]. Circa $1 / 3$ der SCIWORET-Fälle weisen jedoch eine zSKS auf $[5,6]$. In Japan sind mittlerweile sogar $60 \%$ der zRMV-Fälle ältere Patienten mit zSKS ohne Fraktur oder diskoligamentäre Verletzung [7,8]. Die Inzidenz von Hospitalisierungen bei zRMV ist mit begleitender zSKS auf etwa 14 pro 1000 Personenjahre erhöht [9]. Das relative Risiko für eine zRMV bei begleitender Stenose ist zwar schwer detailliert zu bestimmen, aber sicher höher als bei Personen ohne symptomatische zSKS (etwa 5- bis womöglich 125-fach [10-12]). Jedoch ist das absolute Risiko nur sehr gering. Einer Studie von 
Takao et al. folgend ließe sich somit nur bei 0,017\% der Patienten mit zSKS eine zRMV durch eine prophylaktische Dekompression verhindern [12]. Das entspräche einer Number-needed-to-treat von knapp 6000 Eingriffen. Die Häufigkeit perioperativer Morbidität operativer Eingriffe zur Therapie der zervikalen spondylotischen Myelopathie liegt bei etwa $14 \%$ mit einer Mortalität bei 0,3\% [13], was eine Number-needed-to-harm von etwa 7 Eingriffen bedeuten würde.

\section{Diagnostik und Prädiktoren für das Outcome}

Bei der asymptomatischen zSKS stehen also das perioperative Risiko und der Profit (im Sinne der Verhinderung einer zRMV) in einem massiven Missverhältnis zueinander. Somit kann die asymptomatische zSKS auch nicht als signifikanter Negativprädiktor für ein zRMV angenommen werden. Dennoch scheint es ganz offensichtlich Stenosen zu geben, die zu einer zRMV disponieren. Die sichere Identifikation dieser Formen von zSKS mit relevantem negativem Prädiktionspotenzial ist eine der bisher nicht gelösten Aufgaben in der Diagnostik der zSKS.

\section{Merke}

Die Standarddiagnostik für die zSKS ist das MRT.

Sollte ein MRT nicht möglich sein, kann ersatzweise eine Myelografie mit Myelo-CT der HWS helfen, um wenigstens am Abbruch des perimedullären Liquorsaumes eine medulläre Kompression ableiten zu können. Welcher radiologische Parameter sich jedoch am besten eignet, um Fälle mit hohem Gefährdungspotenzial für eine zRMV zu identifizieren, ist unklar. Vielversprechend scheint die Bestimmung des SCOR (Spinal Cord Occupation Ratio) zu sein, mit der die Größenverhältnisse von Myelon und verfügbarem freiem Spinalkanal ins Verhältnis gesetzt werden. Bei einem Cord-Canal-Mismatch von > 70\% (sagittal) oder $80 \%$ (axial) steigt womöglich das Risiko für eine zRMV relevant an [14]. Prospektive randomisierte Studien zu diesem Grenzwert des SCOR liegen aber noch nicht vor. Diskutiert wird zudem, ob sich auffällige 3-T-DTI-Befunde (DTI: Diffusion Tensor Imaging) oder metabolitenfokussierte MRT-Wichtungen sowie bestimmte Biomarker zukünftig in signifikante Korrelation zu einem höheren Risiko der neurologischen Verschlechterung einer zSKS bringen lassen [15].

Nach einem HWS-Trauma ist die Standarddiagnostik zunächst nach aktuellen Empfehlungen vorzunehmen [16, 17]. Bei einer Diskrepanz zwischen neurologischer Ausfallsymptomatik und im CT detektierbarer knöcherner Verletzung empfiehlt sich immer ein HWS-MRT [18]. Dieses zeigt am besten das Ausmaß und die Lokalisation einer evtl. vorliegenden begleitenden Stenose sowie den Umfang einer etwaigen Myelonläsion. Zudem deckt sie am sichersten intraspinale Hämatome, medulläre Ödeme, Bandscheibenherniationen oder sonstige Raumforderungen auf. Die MRT-Befunde haben nicht nur Einfluss auf die Wahl des operativen Verfahrens (s.u.), sondern lassen in gewissen Grenzen wohl auch eine Einschätzung der Prognose nach einer zRMV zu. Die Ausdehnung des Parenchymschadens in der T2-Wichtung, der median-sagittale Durchmesser des Spinalkanals, das Ausmaß der Verlegung des Spinalkanals sowie spezifische radiologische Parameter zur Bestimmung der Stenose (z. B. Pavlov Ratio) haben sich in einzelnen Studien als gute Prognosemarker erwiesen [19-22]. Daneben haben aber auch demografische und neurologische Faktoren (Alter des Patienten, anatomische Höhe der Verletzung, initialer ASIA Score) einen Einfluss auf das Behandlungsergebnis [6].

\section{Therapie}

\section{Indikation und Zeitpunkt einer Operation}

Circa $2 / 3$ der myelopathischen Patienten mit zSKS weisen im zeitlichen Verlauf eine relevante Progression der neurologischen Störungen auf, wenn nicht chirurgisch interveniert wird [23]. Patienten mit zSKS und moderater oder schwerer zervikaler Myelopathie sollten daher einer elektiven OP zugeführt werden. Selbiges gilt für milde Formen der zervikalen Myelopathie, wenn ein strukturierter konservativer Therapiezyklus keine Besserung der Symptome erbringt [24]. Es existiert bisher jedoch kein sicherer Beweis für die positive Wirksamkeit einer dekomprimierenden Operation bei asymptomatischer zervikaler Stenose, sie wird daher (speziell im Sinne einer Prophylaxe einer zRMV) nicht empfohlen [24, 25]. Prophylaktische Dekompressionen werden (nach den oben dargelegten epidemiologischen Maßzahlen) wahrscheinlich auch keinen signifikanten Beitrag zur Reduktion der globalen zRMV-Inzidenz leisten können. Möglicherweise könnte man ein milderes neurologisches Ausfallmuster oder evtl. eine schnellere und umfangreichere Regeneration nach zRMV in der Subgruppe mit vorab dekomprimierter gegenüber der Gruppe mit nicht dekomprimierter symptomatischer zSKS suspizieren [26] - prospektive Studien hierzu gibt es allerdings noch nicht. Trotzdem scheint eine klinische Grauzone zwischen Patienten mit asymptomatischer zSKS und Fällen von zSKS mit Myelopathie zu existieren, die womöglich ein höheres Risiko einerseits für die schleichende Symptomprogredienz der Myelopathie und andererseits für eine akute traumatische zRMV birgt. So wird für nicht myelopathische Fälle mit radiologischen Zeichen der Myelonkompression und klinisch bestehender Radikulopathie sowie elektrophysiologischer Beeinträchtigung der Myelonfunktion (SSEP-Verlängerung) ein höheres Risiko zur Ausbildung einer zervikalen Myelopathie (und evtl. auch einer zRMV) gesehen. 

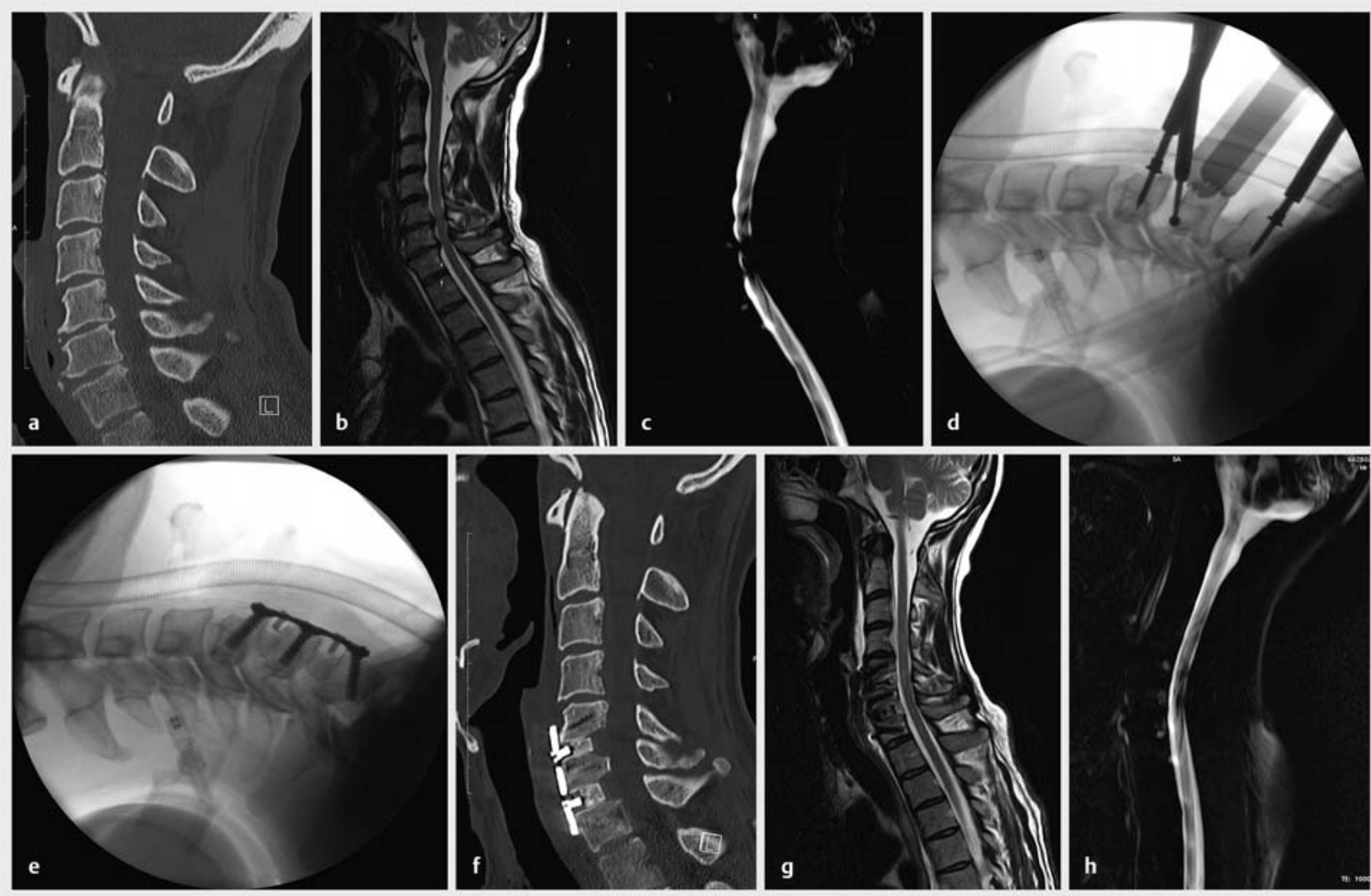

Abb. 1 46-jähriger Mann nach einem Zusammenprall beim Fußball. Kurzzeitige initiale Bewusstlosigkeit und nachfolgend milde armbetonte Tetraparese (ASIA Grad D). Vor dem Trauma seien keine neurologischen Störungen vorhanden gewesen. Im sagittalen HWS-CT (a) sind die degenerativen Veränderungen der Segmente HWK V/VI und HWK VI/VII bereits an den ventralen und dorsalen Spondylophyten erkennbar. Das sagittale HWS-MRT zeigt in den sagittalen T2-Schichten (b) die osteodiskogene Stenose in diesen Höhen sowie den Myelopathieherd oberhalb des Bandscheibenfaches HWK V/VI und den Abbruch des perimedullären Liquorsignals in der Myelosequenz (c). Unter konservativer Therapie mit Zervikalorthese und Kortikosteroiden ergab sich binnen 12 Stunden keine neurologische Besserung. Somit wurde die Indikation zur Dekompression und Fusionierung gestellt. Da die hauptsächliche Raumforderung retrodiskal liegt, reicht hier eine ACDF in den Segmenten HWK V/VI und VI/VII aus. Nach der Diskektomie müssen auch die Retrospondylophyten suffizient abgetragen werden. Das intraoperative seitliche Durchleuchtungsbild (d) zeigt dies exemplarisch mittels 4-mm-Fräse an HWK VI. Abschließend erfolgte eine Segmentfusion mit PEEK-Cages und ventraler Schrauben-Platten-Osteosynthese von HWK V-VII (e). Postoperativ deutliche und rasche Rückbildung der neurologischen Störungen. Das postoperative HWS-CT (f) zeigt die ausreichende Resektion der Retrospondylophyten. Das postoperative HWS-MRT zeigt in der sagittalen T2-Wichtung ( $\mathbf{g}$ ) die ausreichende Dekompression des Halsmarkes und in der Myelosequenz (h) die Wiederherstellung des perimedullären Liquorsignals. Bei Entlassung in die Häuslichkeit nach 5 Tagen postoperativ bestehen nur noch dezente Kribbelparästhesien der linken Hand. Nach 3 Monaten postoperativ ist der Patient beschwerdefrei und ohne neurologische Ausfälle. Das ACDF-Konstrukt ist gut eingeheilt, keine weiteren HWS-Eingriffe notwendig.

Diese Gruppe von Patienten soll unter konservativer Therapie engmaschig kontrolliert und bei Auftreten myelopathischer Symptome frühzeitig operiert werden [23, $24,26]$.

\section{Merke}

Nach einem HWS-Trauma und erfolgtem Ausschluss von Instabilität und akuter intraspinaler Raumforderung kann eine konservative Therapie und engmaschige neurologische Kontrolle insbesondere bei milder Neurologie (ASIA D scheint hier evtl. die Grenze zu sein) indiziert werden.
Es gibt bislang keinen zweifelsfreien Hinweis dafür, dass die neurologischen Ergebnisse im Langzeitverlauf darunter schlechter sind als im Vergleich zu operierten Patienten. Positive Effekte nach Gabe von Methylprednisolon (MP) sind nicht mit hoher Evidenz belegt $[27,28]$. Systematische Reviews fanden aber für die Subgruppe mit MPApplikation innerhalb von 8 Stunden nach Trauma (nicht später) und nur über einen Anwendungszeitraum bis 24 Stunden (nicht länger) bez. einer geringfügigen Verbesserung der motorischen Defizite Hinweise für einen möglichen positiven Einfluss gegenüber dem Komplikationspotenzial von Hochdosis-MP [29]. Die Anwendungen anderer sog. Neuroprotektiva, wie Nimodipin, Riluzol sowie Rho-Enzym-Inhibitoren sind noch nicht hinreichend kli- 

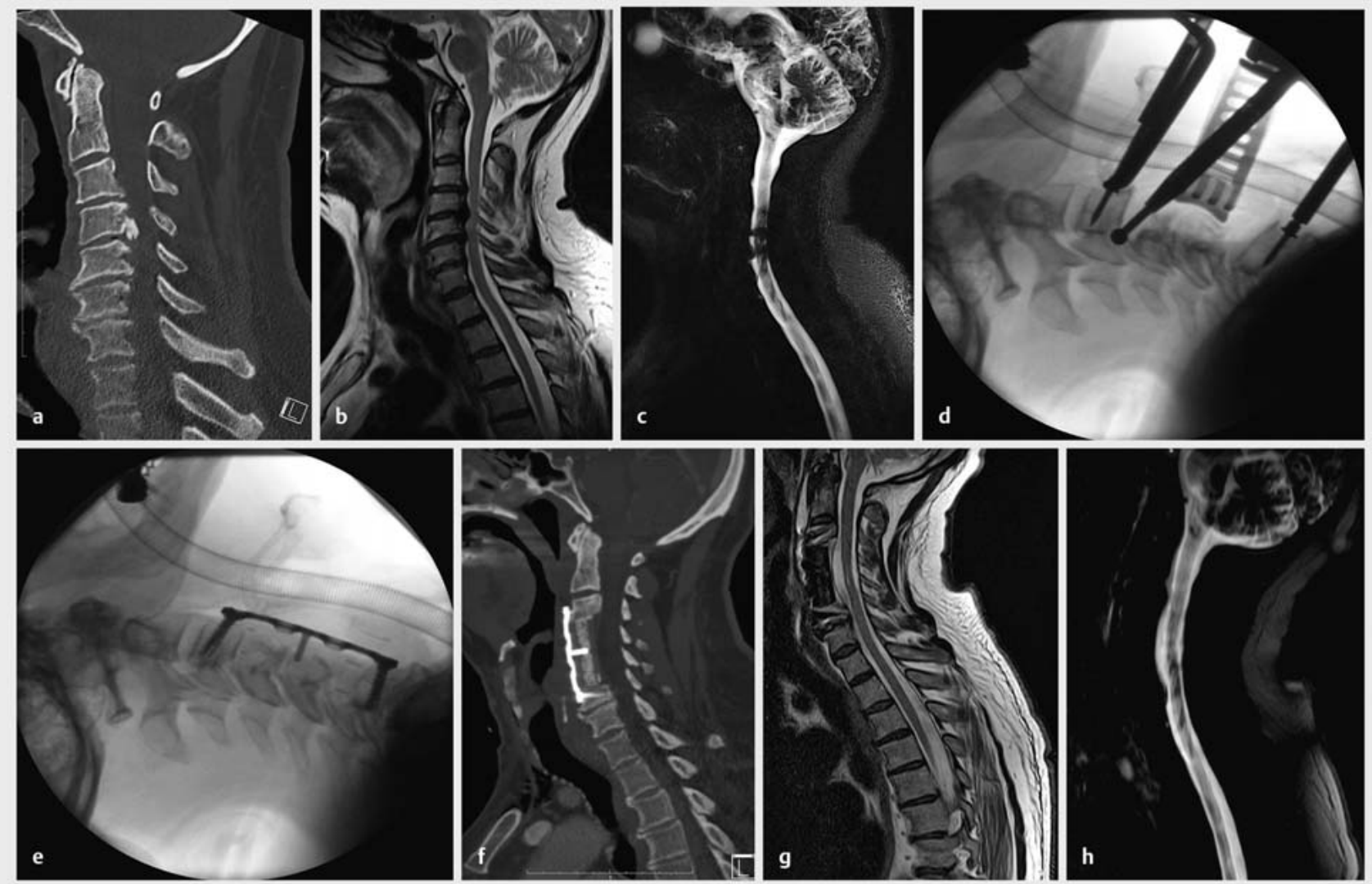

- Abb. 2 78-jähriger Mann nach einem Bagatellstolpersturz im Rahmen einer kardiogenen Synkope. Der Patient sei zuvor mit Werkstattarbeiten an seinem Pkw beschäftigt gewesen und habe sich nach dem Sturz vom Hals abwärts nicht mehr bewegen können. Erst Stunden später wurde er bewegungsunfähig in seiner Werkstatt gefunden. Vor dem Sturz hätten gelegentlich Kribbelparästhesien an den Fingerspitzen bestanden. Einlieferung mit hochgradiger Tetraparese (ASIA Grad B) in eine auswärtige Klinik. Im HWS-CT (a) sind die massiven Spondylophyten erkennbar. Das HWS-MRT zeigt in der sagittalen T2-Wichtung (b) eine mehrsegmentale osteodiskogene Enge von HWK III-VI mit mehreren kleinen Myelopathieherden in allen 3 Segmenten. In der Myelosequenz (c) ist der Abbruch des perimedullären Liquorsaumes dargestellt. Nach bildgebender Primärdiagnostik Beginn einer konservativen Therapie. Nach 1 Woche ergab sich nur eine minimale neurologische Verbesserung, weshalb die Bilder zur konsiliarischen Mitbeurteilung vorgestellt wurden. Angesichts der bildgebenden und neurologischen Befunde wurde OP-Indikation gestellt. Da sich auch eine deutliche Raumforderung retrovertebral hinter HWK IV und V darstellt, wurde eine Korpektomie dieser Wirbel empfohlen. Das intraoperative seitliche Durchleuchtungsbild (d) zeigt exemplarisch die Resektion der Retrospondylophyten mittels 4-mm-Fräse an HWK III. AbschlieBend erfolgte eine Segmentfusion mit einem PEEK-Cage und einer ventralen Schrauben-Platten-Osteosynthese von HWK III-VI (e). Postoperativ erkennbare Rückbildung der neurologischen Störungen. Das postoperative HWS-CT (f) zeigt die ausreichende Resektion der Retrospondylophyten. Das postoperative HWS-MRT zeigt in der sagittalen T2-Wichtung $(\mathbf{g})$ die ausreichende Dekompression des Halsmarkes und in der Myelosequenz (h) die Wiederherstellung des perimedullären Liquorsignals. Nach 10 Tagen postoperativ Verlegung in ein Querschnittzentrum mit einem inkompletten Querschnitt entsprechend ASIA Grad C. Nach 3 Monaten postoperativ ist der Patient wieder frei gehfähig und verspürt nur noch geringfügige sensomotorische Störungen an den Händen (ASIA Grad D). Das ACCF-Konstrukt ist gut eingeheilt, keine weiteren HWS-Eingriffe nötig.

nisch erprobt oder belegt und daher zunächst noch abzulehnen bzw. nur unter spezifischen Studienkonditionen zu unterstützen [30-32]. Wenn eine Besserung unter konservativer Therapie ausreichend schnell $(<24 \mathrm{~h})$ und umfangreich (mindestens 1 ASIA-Grad) eintritt, kann weiter konservativ mit dann wahrscheinlich auch gleich guten Besserungsraten wie mit Operation behandelt werden [33].

Die Wertigkeit einer dekomprimierenden Operation konnte lange Zeit nicht mit hochklassigen Studien in klarer Evidenz über alle Gruppen von zRMV und alle Formen von zSKS hinweg aufgezeigt werden. Mehrere prospektive Einzelstudien und die aktuellen systematischen Reviews weisen mittlerweile aber eindeutig positive Effekte einer operativen Intervention bei der zRMV mit zSKS aus, wenn man bestimmte Subgruppen herausfiltert.

Merke

Sollte eine hinreichend schnelle und umfangreiche Besserung (speziell bei höhergradigen neurologischen Störungen) unter konservativer Therapie ausbleiben, dann sollte möglichst eine Dekompression erfolgen. 
Mag sein, dass die Rate an Patienten mit postoperativer neurologischer Verbesserung nicht immer signifikant verschieden zum konservativen Vorgehen ist. So ist aber häufig wenigstens eine deutlichere oder schnellere neurologische Besserung in den Gruppen mit Operation erkennbar [34-37].

An der primären Myelonschädigung (struktureller primärer Schaden) kann operativ nichts verbessert werden. Das optimale therapeutische (chirurgische) Fenster für das sog. sekundäre Verletzungsstadium (kaskadenartig innerhalb von Minuten bis Stunden durch Ödem, Ischämie, Inflammation und Apoptose ausgelöst [38]) kann aber verpasst werden, wenn mit der OP zu lange gewartet wird. Daher mehren sich Hinweise aus Grundlagenstudien (in vitro [39] und im Tiermodell [40]), dass eine (frühe) chirurgische Therapie relevante Verbesserungen bewirken könnte, und zwar wirksamer als es die konservative Behandlung vermag. Die Ergebnisse früherer klinischer Studien hierzu sind zwar zwiespältig (kontra: [41], pro: [42]). Systematische Reviews und aktuelle Metaanalysen sowie die prospektive STASCIS-Studie favorisieren jedoch heute eindeutig die frühe OP (zwischen 8 und 24 h [34, 36, 43, 44]). Zur sicheren Klärung der Umstände für die Subpopulation von zRMV mit begleitender Stenose und ohne Fraktur sind weitere prospektive und randomisierte Studien notwendig, wie z. B. die OSCIS-Studie (Optimal treatment for Spinal Cord Injury associated with cervical canal Stenosis), die aktuell noch rekrutiert [45]. Insgesamt gibt es derzeit noch keine ausreichende Anzahl an Studien der Evidenzklasse 1 zur Differenzialtherapie oder den optimalen Zeitpunkt einer chirurgischen Therapie des SCI mit Stenose, aber ohne Fraktur, die Aussagen mit hohem Empfehlungsgrad zulassen würden [46].

\section{OP-Verfahren}

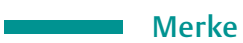

Eine alleinige Osteosynthese ohne suffiziente intraspinale Dekompression des Rückenmarks löst das Problem einer zRMV mit zSKS nicht.

Eine Fusion ist demgegenüber nach ausgedehnter Dekompression nicht selten erforderlich und zur Ruhigstellung des verletzten Myelonabschnittes unserer Meinung nach durchaus sinnvoll. Es müssen also operative Konzepte der Dekompression bei degenerativer zervikaler Myelopathie mit denen der zervikalen Osteosynthese kombiniert werden. Damit wird auch klar, warum diese Spezialfälle in eine Versorgungseinheit mit Expertise in allen ventralen, dorsalen und kombinierten Dekompressions- und Osteosyntheseverfahren gehören.

In der S1-Leitlinie der AWMF aus dem Jahr 2008 (Registernummer 030-052; www.awmf.org/uploads/tx_szleitlinien/030-052_S1) werden hinsichtlich der chirurgischen Zugangswahl bei zervikaler Spinalstenose als wesentliche Entscheidungskriterien die Lokalisation und das Längen- ausmaß der Stenose sowie das sagittale HWS-Profil genannt. Dorsal dominierte Stenosen mit erhaltener zervikaler Gesamtlordose sollten demnach eher von dorsal operiert werden. Für Stenosen ventraler Genese über maximal 3 Segmente bei kyphotischer Stellung sollen hingegen anteriore Techniken bevorzugt werden $[47,48]$. Sofern die Stenosierung des Zervikalkanals nicht nur begrenzt retrodiskal liegt (in solchen Fällen wird man eine mehrsegmentale ACDF indizieren), sondern auch retrovertebral (in diesen Fällen kann die Stenosierung über das Bandscheibenfach häufig nicht ausreichend abgetragen werden), kommt als Dekompressionsverfahren am besten die Korpektomie mit anschließender Fusion in Betracht [49]. Nach der anterioren spinalen Dekompression ist eine ventrale Segmentfusion geboten. Diese kann als intervertebrale Fusion (ACDF, > Abb. 1), als Wirbelkörperersatz (ACCF, - Abb. 2) oder einer Kombinationen beider Verfahren (sog. Hybridfusion) erfolgen. Es kristallisiert sich zunehmend heraus, dass autologer Knochen nicht mehr als alleiniger „Goldstandard“ der ventralen Zervikalfusion zu bezeichnen ist. So gaben in einer weltweiten Umfrage unter AO-Spine-Mitgliedern zur ACDF $64 \%$ der Operateure an, einen Cage zur Fusion zu verwenden, nur $20 \%$ votierten für autologen Knochenspan [50]. Eine Umfrage unter deutschen und österreichischen Wirbelsäulenoperateuren zur ACCF zeigte ähnliche Tendenzen. So nutzen nur noch etwa $16 \%$ der Operateure autologen Knochen (Beckenkamm oder Fibula) zur Rekonstruktion von Halswirbelkörpern, während $84 \%$ der Wirbelsäulenchirurgen unterschiedliche Cages verwenden [51]. Die weit überwiegende Mehrzahl der aktuellen Publikationen zu dieser Thematik beschreibt mittlerweile vergleichbar gute klinische und radiologische Ergebnisse für die Zervikal-Cages - aber eben ohne die zusätzliche Entnahmemorbidität bei Knochenautograft. Neben den metallbasierten Cage-Materialien (heutzutage fast ausschließlich Titan) werden seit einigen Jahren zunehmend auch gleich gut geeignete Kunststoff-Cages zur ACDF und ACCF verwendet ( $\vee$ Abb. 1 und $\triangleright$ Abb. 2 ), deren radiologische Artefaktbildung jedoch geringer als bei Metall-Cages ausfällt $[52,53]$.

\section{Merke}

Eine zusätzliche ventrale Plattenosteosynthese ist auch nach monosegmentaler Diskektomie bei der zSKS mit zRMV eher empfehlenswert, nach Korpektomie ist sie sogar als Standard zu bezeichnen.

Indikationen für den dorsalen Zugang bestehen, wenn längerstreckige Stenosen (>3 Segmente, insbesondere bei lordotischer HWS-Gesamtstellung) vorliegen oder das kompressive Element seinen Ursprung überwiegend von dorsal aus nimmt $[49,54]$. Insbesondere multisegmentale Laminektomien sollten dann aber durch eine Osteosynthese und Fusion ergänzt werden, um eine Spätdeformität zu vermeiden [55]. Am weitesten verbreitet dürfte die Osteosynthese mittels Massa-lateralis-Schrau- 
ben sein. Eine Pedikelschraubenosteosynthese ist zwar resistenter hinsichtlich Schraubenausriss, gleichzeitig ist sie aber auch etwas komplikationsträchtiger (insbesondere in den Händen des Ungeübten) sowie aufwendiger (da meist eine intraoperative Navigation zur Anwendung kommen dürfte). Laminaschrauben sind wegen fehlender oder teilresezierter Lamina(e) meist nicht sinnvoll anzuwenden. Auch die dorsalen Dekompressionen selbst haben in der Vergangenheit strukturerhaltende Verfeinerungen/Miniaturisierungen erfahren. Prozeduren, wie bspw. die Laminoplastie, haben sich gegenüber der Laminektomie jedoch bisher nicht als signifikant vorteilhaft erwiesen [56]. Die mikrochirurgische Dekompression über interlaminäre Fensterungen oder Hemilaminektomien unter Funktionserhalt des dorsalen Band- und Gelenkapparates stellt für degenerative Zervikalstenosen ebenfalls eine fusionslose minimalinvasive Behandlungsoption dar [57].

Wenn die Indikationsstellung für ein geeignetes isoliertes ventrales oder dorsales Dekompressionsverfahren korrekt war und eine zusätzliche Osteosynthese nach der Dekompression erfolgt ist, so ergeben sich im Regelfall zumeist keine Indikationen für kombinierte (360 ${ }^{\circ}$ ) Verfahren. Bei insuffizienter Dekompression oder posttraumatischer Deformität und bei bestehendem (oder zu erwartendem) Materialversagen (speziell bei langstreckiger Fusion) kann ein zusätzlicher Eingriff von der Gegenseite aber gelegentlich notwendig werden. Dies ist besonders bei Fällen mit Tetraparese (ohne suffiziente Rumpfkontrolle) u.U. sinnvoll, da diese Patienten häufig unkoordiniert Gleichgewichtskompensationsmanöver mit dem Kopf vornehmen und bei passiven Mobilisationen nicht selten an deren Kopf bzw. Hals angesetzt wird.

\section{Duraerweiterung nach SCI}

Der optimale Spinal Cord Perfusion Pressure (SCPP) liegt bei $90-100 \mathrm{mmHg}$. Der intraspinale Druck (ISP) liegt nach einer zRMV erfahrungsgemäß bei $20-40 \mathrm{mmHg}$. Der MAP (mittlerer arterieller Druck) sollte daher bei zRMV über 110-130 mmHg gehalten werden [58]. Eine Messung des ISP macht dabei nur auf der Höhe der Verletzung Sinn. Der intrathekale Druck an der LWS ist bspw. zur Bestimmung von ISP und SCPP auf Höhe eines verletzten Zervikalsegmentes insuffizient. Allenfalls kann anhand nachweisbarer Liquorpulsationswellen ein indirekter Hinweis für eine ausreichende Dekompression gewonnen werden. Die (weniger invasive) subdurale und die intraparenchymatöse spinale Druckmessung zeigen vergleichbare Werte [59]. Für die intraspinale Druckmessung und ein gezieltes ISP- und SCPP-Management (ISP $<10 \mathrm{mmHg}$; SCPP > $90 \mathrm{mmHg}$ ) ergeben sich nach systematischem Review bereits erste Hinweise für ein besseres neurologisches Ergebnis nach Rückenmarkverletzung $[60,61]$. Auch ließ sich darunter eine Amplitudensteigerung von MEP und per Mikrodialyse eine 3-fach höhere Gewebepenetration von Dexamethason, eine bessere
Versorgung mit Stoffwechselprodukten und weniger Anfall von toxisch-ischämischen Metaboliten zeigen [62].

Durch eine isolierte Laminektomie kann der intradurale Druck nicht effektiv gesenkt und der SCPP nicht effektiv gesteigert werden. Mittels zusätzlicher Durotomie und ggf. Duraerweiterungsplastik kann dies jedoch bewerkstelligt werden. Nach einem systematischen Review wurden randomisierte Studien gefordert, die den Einfluss von Duraerweiterungen auf das neurologische Ergebnis nach einer Myelonläsion untersuchen sollen, da aktuell nicht genügend Daten vorliegen, die eine Entlastungsdurotomie am Rückenmark mit starkem Empfehlungsgrad unterstützen [61].

\section{Gutachterliche Aspekte}

Einer zRMV geht in aller Regel ein Unfallereignis voraus. Sofern vor dem Unfall Beschwerdefreiheit bestanden hat (Diagnosemitteilungen der Krankenkasse können hier informativ sein), gilt es zunächst, die Kausalität zu klären. Ist ein dokumentiertes Unfallereignis überhaupt geeignet, den nachweisbaren Schaden zu verursachen? Es ist heute akzeptiert, dass Geschwindigkeitsveränderungen $\delta v<10-15 \mathrm{~km} / \mathrm{h}$ am ehesten nicht in der Lage sein dürften, relevante strukturelle Schäden an der HWS (wie Frakturen oder diskoligamentäre Rupturen) zu bewirken [63]. Wie oben geschildert, ist bei einer vorbestehenden zSKS eine gewisse (individuelle) Prädisposition für eine zRMV gegeben. Somit kann nicht ausgeschlossen werden, dass derart Betroffene schon bei geringeren Bewegungsmomenten und auch ohne Nachweis von Frakturen und Bandrupturen eine relevante Halsmarktangierung erlitten haben könnten (Stichwort: SCIWORET).

Bei vorbestehender/begleitender zSKS ergibt sich, neben der schwierigen Klärung der Kausalitätsfragen, zudem die nicht weniger komplexe Problematik der Einschätzung der überwiegenden Verursachung einer zRMV (Unfall vs. Vorerkrankung). Bei vorbestehender symptomatischer zSKS, die sich durch ein minimalstes Unfallereignis nicht oder nur marginal verändert hat, wird man eher zu dem Schluss kommen, dass die überwiegende Ursache die vorbestehende Degeneration sein dürfte. Bei nachweislich prätraumatisch asymptomatischer zSKS mit einem relevanten Unfallereignis und nachfolgend höhergradiger zRMV, kann man hingegen eher eine Hauptverursachung durch den Unfall suspizieren. Die Schwierigkeit liegt sicher nicht in der Entscheidung zwischen diesen beiden geschilderten Extremsituationen, sondern in der Beurteilung von (womöglich häufiger auftretenden) Fällen aus der Grauzone dazwischen (also z. B. bei relevanter Vorschädigung kombiniert mit relevantem Unfallereignis).

Da speziell in Deutschland eine zunehmende Neigung zur versicherungsrechtlichen und auch gerichtlichen Verhandlung von HWS-Distorsionen zu verzeichnen ist, 
kommt insbesondere der initialen Befundung eine erhebliche Bedeutung zu. Je genauer der allgemeine körperliche Status und je detaillierter eine neurologische Grunduntersuchung nach einem Unfall mit vermeintlicher HWS-Beteiligung ausfallen, umso sicherer kann auch zeitversetzt noch eine gerechte abschließende Beurteilung getroffen werden.

\section{Merke}

In diesem Zusammenhang empfehlen sich (bereits in Erwartung zukünftiger gutachterlicher Fragen) eine möglichst lückenlose Befunddokumentation (nicht nur initial, sondern auch im Verlauf nach dem Unfall) sowie eine suffiziente Untersuchung, ggf. sollte eine neurophysiologische Untersuchung zur Befundobjektivierung inkludiert werden.

Sofern operative Eingriffe erfolgen, sollten auffällige Veränderungen (wie z.B. eingeblutete Muskeln, gerissene Bänder etc.) intraoperativ fotodokumentiert und/oder im OP-Bericht eindeutig niedergelegt werden. Zudem empfiehlt sich möglichst die Einsendung von Material zur histologischen Begutachtung auf erfolgte Einblutungen.

\section{Interessenkonflikt}

Die Autoren erklären, dass kein Interessenkonflikt vorliegt.

\section{Autorinnen/Autoren}

\section{Chris Schulz}

Dr. med., Stellv. Klinischer Direktor, Klinik für Neurochirurgie, Bundeswehrkrankenhaus Ulm

\section{René Mathieu}

Dr. med., Oberarzt, Klinik für Neurochirurgie, Bundeswehrkrankenhaus Ulm

\section{Uwe Max Mauer}

Prof. Dr., Klinischer Direktor der Klinik für Neurochirurgie, Bundeswehrkrankenhaus Ulm

\section{Korrespondenzadresse}

\section{Prof. Dr. Uwe Max Mauer}

Klinik für Neurochirurgie

Bundeswehrkrankenhaus Ulm

Oberer Eselsberg 40

$89081 \mathrm{Ulm}$

Tel.: $0731 / 1710-2201$

Fax: 0731/17 10-2205

UweMaxMauer@bundeswehr.org

\section{Literatur}

[1] Schnake KJ, Schroeder GD, Vaccaro AR et al. AOSpine classification systems (subaxial, thoracolumbar). J Orthop Trauma 2017; 31 (Suppl. 4): S14-S23

[2] Chang V, Ellingson BM, Salamon $\mathrm{N}$ et al. The risk of acute spinal cord injury after minor trauma in patients with preexisting cervical stenosis. Neurosurgery 2015; 77: 561-565

[3] Stein DM, Kufera JA, Ho SM et al. Occupant and crash characteristics for case occupants with cervical spine injuries sustained in motor vehicle collisions. J Trauma 2011; 70: 299-309

[4] Tewari MK, Gifti DS, Singh P et al. Diagnosis and prognostication of adult spinal cord injury without radiographic abnormality using magnetic resonance imaging: analysis of 40 patients. Surg Neurol 2005; 63: 204-209

[5] Kato H, Kimura A, Sasaki R et al. Cervical spinal cord injury without bony injury: a multicenter retrospective study of emergency and critical care centers in Japan. J Trauma 2008; 65: 373-379

[6] Aarabi B, Alexander M, Mirvis SE et al. Predictors of outcome in acute traumatic central cord syndrome due to spinal stenosis. J Neurosurg Spine 2011; 14: 122-130

[7] Koyanagi I, Iwasaki Y, Hida K et al. Acute cervical cord injury without fracture or dislocation of the spinal column. J Neurosurg 2000; 93: 15-20

[8] Chikuda H, Yasunaga H, Takeshita K et al. Mortality and morbidity after high-dose methylprednisolone treatment in patients with acute cervical spinal cord injury: a propensitymatched analysis using a nationwide administrative database. Emerg Med ] 2014; 31: 201-206

[9] Tetreault LA, Karadimas S, Wilson JR et al. The natural history of degenerative cervical myelopathy and the rate of hospitalization following spinal cord injury: an updated systematic review. Global Spine J 2017; 7 (3 Suppl.): 28S-34S

[10] Wu JC, Chen YC, Liu L et al. Conservatively treated ossification of the posterior longitudinal ligament increases the risk of spinal cord injury: a nationwide cohort study. J Neurotrauma 2012; 29: 462-468

[11] Wu JC, Ko CC, Yen YS et al. Epidemiology of cervical spondylotic myelopathy and its risk of causing spinal cord injury: a national cohort study. Neurosurg Focus 2013; 35: E10

[12] Takao T, Morishita Y, Okada S et al. Clinical relationship between cervical spinal canal stenosis and traumatic cervical spinal cord injury without major fracture or dislocation. Eur Spine J 2013; 22: 2228-2231

[13] Fehlings MG, Tetreault LA, Kurpad S et al. Change in functional impairment, disability, and quality of life following operative treatment for degenerative cervical myelopathy: a systematic review and meta-analysis. Global Spine J 2017; 7 (3 Suppl.): 53S-69S

[14] Nouri A, Montejo J, Sun X et al. Cervical cord-canal mismatch: a new method for identifying predisposition to spinal cord injury. World Neurosurg 2017; 108: 112-117

[15] Ganau M, Holly LT, Mizuno J et al. Future directions and new technologies for the management of degenerative cervical myelopathy. Neurosurg Clin N Am 2018; 29: 185-193

[16] Schleicher P, Scholz M, Kandziora F et al. Therapieempfehlungen zur Versorgung von Verletzungen der subaxialen Halswirbelsäule. Z Orthop Unfall 2017; 155: 556-566

[17] Fehlings MG, Tetreault LA, Wilson JR et al. A clinical practice guideline for the management of acute spinal cord injury: introduction, rationale, and scope. Global Spine J 2017; 7 (3 Suppl.): 84 S-94S 
[18] Fehlings MG, Martin AR, Tetreault LA et al. A clinical practice guideline for the management of patients with acute spinal cord injury: recommendations on the role of baseline magnetic resonance imaging in clinical decision making and outcome prediction. Global Spine J 2017; 7 (3 Suppl.): 221S-230S

[19] Boldin C, Raith J, Fankhauser F et al. Predicting neurologic recovery in cervical spinal cord injury with postoperative MR imaging. Spine (Phila Pa 1976) 2006; 31: 554-559

[20] Song KJ, Choi BW, Kim S] et al. The relationship between spinal stenosis and neurological outcome in traumatic cervical spine injury: an analysis using Pavlov's ratio, spinal cord area, and spinal canal area. Clin Orthop Surg 2009; 1: 11-18

[21] Wilson JR, Arnold PM, Singh A et al. Clinical prediction model for acute inpatient complications after traumatic cervical spinal cord injury: a subanalysis from the Surgical Timing in Acute Spinal Cord Injury Study. J Neurosurg Spine 2012; 17: 46-51

[22] Kurpad S, Martin AR, Tetreault LA et al. Impact of baseline magnetic resonance imaging on neurologic, functional, and safety outcomes in patients with acute traumatic spinal cord injury. Global Spine J 2017; 7 (3 Suppl.): 151S-174S

[23] Rhee J, Tetreault LA, Chapman JR et al. Nonoperative versus operative management for the treatment degenerative cervical myelopathy: an updated systematic review. Global Spine J 2017; 7 (3 Suppl.): 35S-41S

[24] Fehlings MG, Tetreault LA, Riew KD et al. A clinical practice guideline for the management of patients with degenerative cervical myelopathy: recommendations for patients with mild, moderate, and severe disease and nonmyelopathic patients with evidence of cord compression. Global Spine J 2017; 7 (3 Suppl.): 70S-83S

[25] Takao T, Okada S, Morishita Y et al. Clinical influence of cervical spinal canal stenosis on neurological outcome after traumatic cervical spinal cord injury without major fracture or dislocation. Asian Spine J 2016; 10: 536-542

[26] Chen LF, Tu TH, Chen YC et al. Risk of spinal cord injury in patients with cervical spondylotic myelopathy and ossification of posterior longitudinal ligament: a national cohort study. Neurosurg Focus 2016; 40: E4

[27] Fehlings MG, Wilson JR, Tetreault LA et al. A clinical practice guideline for the management of patients with acute spinal cord injury: recommendations on the use of methylprednisolone sodium succinate. Global Spine J 2017; 7 (3 Suppl.): 2035-211S

[28] Pointillart V, Petitjean ME, Wiart L et al. Pharmacological therapy of spinal cord injury during the acute phase. Spinal Cord 2000; 38: 71-76

[29] Fehlings MG, Wilson JR, Harrop JS et al. Efficacy and safety of methylprednisolone sodium succinate in acute spinal cord injury: a systematic review. Global Spine J 2017; 7 (3 Suppl.): $1165-1375$

[30] Jia YF, Gao HL, Ma LJ et al. Effect of nimodipine on rat spinal cord injury. Genet Mol Res 2015; 14: 1269-1276

[31] Fehlings MG, Kopjar B, Grossman RG. 329 Efficacy and safety of riluzole in acute spinal cord injury: rationale and design of aospine phase III multicenter double-blinded randomized controlled trial (RISCIS). Neurosurgery 2016; 63 (Suppl. 1): S196

[32] Fehlings MG, Kim KD, Aarabi B et al. Rho inhibitor VX-210 in acute traumatic subaxial cervical spinal cord injury: Design of the SPinal cord injury Rho INhibition investiGation (SPRING) clinical trial. J Neurotrauma 2018. doi:10.1089/ neu.2017.5434

[33] Kawano O, Ueta T, Shiba K et al. Outcome of decompression surgery for cervical spinal cord injury without bone and disc injury in patients with spinal cord compression: a multicenter prospective study. Spinal Cord 2010; 48: 548-553

[34] La Rosa G, Conti A, Cardali S et al. Does early decompression improve neurological outcome of spinal cord injured patients? Appraisal of the literature using a meta-analytical approach. Spinal Cord 2004; 42: 503-512

[35] Chikuda H, Seichi A, Takeshita K et al. Acute cervical spinal cord injury complicated by preexisting ossification of the posterior longitudinal ligament: a multicenter study. Spine (Phila Pa 1976) 2011; 36: 1453-1458

[36] Fehlings MG, Tetreault LA, Wilson JR et al. A clinical practice guideline for the management of patients with acute spinal cord injury and central cord syndrome: recommendations on the timing ( $\leq 24$ hours versus $>24$ hours) of decompressive surgery. Global Spine J 2017; 7 (3 Suppl.): 195S-202S

[37] Wilson JR, Tetreault LA, Kwon BK et al. Timing of decompression in patients with acute spinal cord injury: a systematic review. Global Spine J 2017; 7 (3 Suppl.): 95S-115S

[38] Ditunno JF, Little JW, Tessler A et al. Spinal shock revisited: a four-phase model. Spinal Cord 2004; 42: 383-395

[39] Carlson GD, Gorden CD, Oliff HS et al. Sustained spinal cord compression: part I: time-dependent effect on long-term pathophysiology. J Bone Joint Surg Am 2003; 85-A: 86-94

[40] Dimar JR 2nd, Glassman SD, Raque GH. The influence of spinal canal narrowing and timing of decompression on neurologic recovery after spinal cord contusion in a rat model. Spine (Phila Pa 1976) 1999; 24: 1623-1633

[41] Vaccaro AR, Daugherty RJ, Sheehan TP et al. Neurologic outcome of early versus late surgery for cervical spinal cord injury. Spine (Phila Pa 1976) 1997; 22: 2609-2613

[42] Chen TY, Dickman CA, Eleraky M et al. The role of decompression for acute incomplete cervical spinal cord injury in cervical spondylosis. Spine (Phila Pa 1976) 1998; 23: 2398-2403

[43] Lenehan B, Fisher CG, Vaccaro A et al. The urgency of surgical decompression in acute central cord injuries with spondylosis and without instability. Spine (Phila Pa 1976) 2010; 35 (21 Suppl.): S180-S186

[44] Fehlings MG, Vaccaro A, Wilson JR et al. Early versus delayed decompression for traumatic cervical spinal cord injury: results of the Surgical Timing in Acute Spinal Cord Injury Study (STASCIS). PLoS One 2012; 7: e32037

[45] Chikuda H, Ohtsu H, Ogata T et al. Optimal treatment for spinal cord injury associated with cervical canal stenosis (OSCIS): a study protocol for a randomized controlled trial comparing early versus delayed surgery. Trials 2013; 14: 245

[46] Aarabi B, Hadley MN, Dhall SS et al. Management of acute traumatic central cord syndrome (ATCCS). Neurosurgery 2013; 72 (Suppl. 2): S195-S204

[47] Rao RD, Gourab K, David KS. Operative treatment of cervical spondylotic myelopathy. J Bone Joint Surg Am 2006; 88: 1619-1640

[48] Ghogawala Z. Anterior cervical option to manage degenerative cervical myelopathy. Neurosurg Clin N Am 2018; 29: 8389

[49] Mummaneni PV, Kaiser MG, Matz PG Cervical surgical techniques for the treatment of cervical spondylotic myelopathy. J Neurosurg Spine 2009; 11: 130-141

[50] Yoon ST, Konopka JA, Wang JC et al. ACDF graft selection by surgeons: survey of AOSpine members. Global Spine J 2017; 7: 410-416

[51] Hartmann S, Tschugg A, Obernauer J Cervical corpectomies: results of a survey and review of the literature on diagnosis, 
indications, and surgical technique. Acta Neurochir (Wien) 2016; 158: 1859-1867

[52] Kersten RF, van Gaalen SM, de Gast A et al. Polyetheretherketone (PEEK) cages in cervical applications: a systematic review. Spine J 2015; 15: 1446-1460

[53] Schulz C, Mauer UM, Mathieu R. Implantatassoziierte Komplikationen sowie klinischer und radiologischer Verlauf nach anteriorer zervikaler Korpektomie und Cage-Fusion - retrospektiver Vergleich von PEEK- gegen Titan-Cages. Z Orthop Unfall 2017; 155: 201-208

[54] Meyer F, Börm W, Thomé C. Degenerative cervical spinal stenosis: current strategies in diagnosis and treatment. Dtsch Arztebl Int 2008; 105: 366-372

[55] Lawrence BD, Brodke DS. Posterior surgery for cervical myelopathy: indications, techniques, and outcomes. Orthop Clin North Am 2012; 43: 29-40

[56] Yoon ST, Hashimoto RE, Raich A et al. Outcomes after laminoplasty compared with laminectomy and fusion in patients with cervical myelopathy: a systematic review. Spine (Phila Pa 1976) 2013; 38 (22 Suppl. 1): S183-S194

[57] Schulz C, Kunz U, Mauer UM et al. Selektive dorsale Dekompression der degenerativen Zervikalstenose. Orthopäde 2014; 43: $568-574$

[58] Varsos GV, Werndle MC, Czosnyka ZH et al. Intraspinal pressure and spinal cord perfusion pressure after spinal cord injury: an observational study. J Neurosurg Spine 2015; 23: 763-771
[59] Phang I, Zoumprouli A, Saadoun S et al. Safety profile and probe placement accuracy of intraspinal pressure monitoring for traumatic spinal cord injury: Injured Spinal Cord Pressure Evaluation study. J Neurosurg Spine 2016; 25: 398-405

[60] Saadoun S, Chen S, Papadopoulos MC. Intraspinal pressure and spinal cord perfusion pressure predict neurological outcome after traumatic spinal cord injury. J Neurol Neurosurg Psychiatry 2017; 88: 452-453

[61] Tykocki T, Poniatowski Ł, Czyż M et al. Intraspinal pressure monitoring and extensive duroplasty in the acute phase of traumatic spinal cord injury: a systematic review. World Neurosurg 2017; 105: 145-152

[62] Phang I, Zoumprouli A, Papadopoulos MC et al. Microdialysis to optimize cord perfusion and drug delivery in spinal cord injury. Ann Neurol 2016; 80: 522-531

[63] Elbel M, Kramer M, Huber-Lang $M$ et al. Deceleration during 'real life' motor vehicle collisions - a sensitive predictor for the risk of sustaining a cervical spine injury? Patient Saf Surg 2009; $3: 5$

Bibliografie

DOI https://doi.org/10.1055/a-0574-3131

OP-JOURNAL 2018; 34: 118-126 @ Georg Thieme Verlag KG Stuttgart · New York ISSN 0178-1715 